\title{
Aprendizaje Basado en Proyectos para el desarrollo de las competencias profesionales del maestro: una propuesta de innovación docente desde la Didáctica de las Ciencias Sociales
}

\section{Project Based Learning to develop teacher's professional competences: an innovation teaching proposal in Didactic of Social Sciences}

\author{
Esther López Torres \\ Departamento de Didáctica de las Ciencias \\ Experimentales, Sociales y de la Matemática \\ Universidad de Valladolid
}

Resumen: En este trabajo presentamos una propuesta de innovación docente desde la Didáctica de las Ciencias Sociales que se imparte en las aulas universitarias del Grado de Primaria basada en la aplicación de la metodología de Aprendizaje Basado en Problemas (ABP). Después de comentar brevemente el contexto teórico en el que se enmarca y, condicionados por éste, los objetivos que nos planteamos alcanzar, se detallan los elementos que definen nuestra propuesta explicando el planteamiento del proyecto que pedimos a los alumnos, los criterios de calidad y el seguimiento del mismo, para pasar después al análisis y valoración de los resultados preliminares de su desarrollo, en los que apoyaremos nuestras primeras conclusiones.

Palabras clave: innovación docente universitaria, Didáctica de las Ciencias Sociales, aprendizaje basado en problemas, proyecto didáctico, Educación Primaria.

Abstract: In this paper we present a proposal for teaching innovation from the Didactic of Social Sciences that is taught in university classrooms Grade Primary Education based on the application of the methodology of Problem Based Learning (PBL). After discussing briefly the theoretical context in which it occurs and, conditional on this, the goals we wish to achieve, we detail the elements that define our proposal explaining the project approach that we ask to students, quality criteria and their follow-up. Then we move on to the analysis and evaluation of the preliminary results of its development, which will support our initial findings.

Keywords: university teaching innovation, Didactic of Social Sciences, problem based learning, didactic project, Elementary Education.

(Fecha de recepción: mayo, 2014, y de aceptación: octubre, 2014)

DOI: 10.7203/DCES.29.3775 


\section{Contexto teórico: EEES, realidad en las aulas y proyectos de renovación}

\author{
El EEES: Una apuesta por \\ metodologías de enseñanza más \\ activas
}

En las aulas universitarias del Grado de Maestro, tanto de Infantil como de Primaria, insistimos a nuestros alumnos, y desde diferentes áreas, en la importancia de tener en cuenta los intereses de los escolares para generar en las aulas aprendizajes significativos. De un modo más o menos explícito, criticamos los modelos de enseñanza puramente expositivos y/o basados en la utilización del libro de texto como único guía para seleccionar los contenidos y plantear actividades. Frente a este modelo tratamos de abordar, desde diferentes frentes, nuestra apuesta por formar maestros que desarrollen metodologías más activas, más centradas en el alumno y en el proceso y menos en el profesor y los resultados, y al mismo tiempo más coherente con la estructura competencial que en la enseñanza básica proponen los currícula oficiales.

Asumiendo así los elementos comunes que, como señala Rosario Cubero, subyacen a las distintas orientaciones constructivistas, y que se centran en "una concepción de las personas como agentes activos y una interpretación de la construcción del conocimiento como un proceso social y situado en un contexto cultural e histórico" (Cubero, 2005 , p. 44), apostamos por un modelo que invita a un tratamiento más globalizador e interdisciplinar de los contenidos para generar en el discente un aprendizaje más lógico y real, al tiempo que más eficaz (Gavaldà y Pons i Altés, 2010). En dicha estructura, encajan a la perfección metodologías inductivas como pueden ser el trabajo por proyectos o centrado en casos o problemas, descrito y expuesto por Kilpatrick ya en 1918, por cuanto es "un método de carácter globalizador, apoyado en las ideas e intereses del alumnado, basado en situaciones reales y con un marcado sentido social" (Pozuelos, 2007, p. 15). Este método de trabajo en el aula no se identifica con una corriente estricta sino que "toma en consideración aspectos relativos al proceso de aprendizaje (constructivismo); al intercambio y el apoyo como base para la construcción de los significados y el establecimiento las relaciones sociales y de convivencia (enfoque cooperativo); la integración de los contenidos y el reconocimiento de distintas formas de saber para interpretar la complejidad de la cultura actual (currículum integrado); la investigación como proceso de búsqueda que conduce a la asimilación significativa de las habilidades, procedimientos y actitudes que se pretenden desarrollar (aprender investigando); la necesidad de hacer del conocimiento un objeto al servicio de la mejora de la realidad en la que se vive (perspectiva crítica) e implicar en esta tarea a toda la comunidad de forma que aprender se constituya en una acción social y abierta (orientación comunitaria)" (Ibidem, p. 26). 
Sin duda alguna, estas metodologías activas, también pueden y deben ser, y de hecho lo están siendo, aplicadas en las aulas universitarias como respuesta a la filosofía del Espacio Europeo de Educación Superior (Solaz-Portolés et al., 2011), centrada fundamentalmente en el proceso de aprendizaje. El sistema de créditos ECTS (European Credit Transfer System) que ha impuesto el proceso de convergencia europea supone de hecho una nueva manera de ver la formación universitaria al centrar la atención, no solamente en la enseñanza, sino también, y sobre todo, y admitiendo con ello las teorías constructivistas, "en el protagonismo del alumnado, viéndolo como la persona que tiene que construir su aprendizaje, mediante el esfuerzo y la implicación activa en el propio proceso de aprender" (Parcerisa, 2006, p. 52) para adquirir unas competencias profesionales. En el EEES se persigue, ante todo, que nuestros estudiantes desarrollen aptitudes para la "creatividad, la flexibilidad, la capacidad de adaptación y la habilidad de aprender a aprender y a resolver problemas, durante toda la vida" (Parcerisa, 2006, p. 54), lo cual exige a los profesores una revisión de la práctica docente y una apuesta por metodologías de enseñanza más activas (Prieto et al., 2006), y más aún en las Facultades de Educación (Egido et al., 2007).

Las distintas experiencias de aprendizaje basado en problemas (ABP), conocido en su versión inglesa como Problem Based Learnig (PBL), y su variante, Aprendizaje Basado en Pro- yectos, en las aulas universitarias, inicialmente en las titulaciones de la rama sanitaria y las técnicas (medicina, biología, arquitectura, ingeniería, informática...) y progresivamente en estudios sociales, demuestran que estos métodos de aprendizaje mejoran la motivación del alumnado, haciendo que se esfuerce más (Garrigós y Valero, 2012); favoreciendo su desarrollo intelectual y el aprendizaje (Ramsden, 2003; Mabrouk, 2007) y capacitándolo para encontrar la solución de problemas, comprender conceptos complejos, desarrollar habilidades para aplicar el conocimiento así como estrategias de razonamiento y metacognitivas, y para trabajar en equipo (Barret, 2005; Prince y Felder, 2006). En definitiva, este tipo de metodologías contribuyen al desarrollo de habilidades transversales que luego son apreciadas en el momento del ejercicio profesional (Solaz-Portolés et al., 2011, pp. 182-183).

\section{Resistencias: realidad escolar, preconceptos del alumno $y$ contradicciones internas en torno al modelo ideal de enseñanza}

Cuando en las aulas del Grado de Maestro tratamos de promover en nuestro alumnado su apuesta por metodologías de eseñanza activa nos encotramos con una serie de resistencias que proceden, en muchas ocasiones, del ámbito escolar (Seva et al., 2010), el cual actúa en ellos como un bloqueo a esa transformación y por doble vía: por un lado, en su socialización preprofesional duran- 
te las prácticas; por otro en su propia socialización como escolares.

Como es evidente, nuestros estudiantes, que se preparan para ser maestros, llegan a conocer con bastante grado de profundidad las teorías que, desde hace más de tres décadas, fundamentan estas metodologías de enseñanza que convierten al alumno en protagonista del proceso y que a través de la investigación-acción fomentan el aprendizaje por descubrimiento. Sin embargo su contacto con la realidad escolar ya durante su proceso formativo genera el primer problema para que estas metodologías "calen" de verdad en la configuración de su identidad como profesional de la enseñanza.

Cuando realizan sus propias Prácticas dentro del Grado, con demasiada frecuencia se ven imposibilitados para aplicar y experimentar metodologías activas porque sus maestros tutores suelen ceñirse, y por ende también nuestros alumnos en prácticas, al método que propone una editorial, restando protagonismo a los escolares en su propio proceso de aprendizaje. Esto en ocasiones genera una cierta tensión entre los profesores universitarios y nuestros alumnos, que se quejan de la "desconexión" que existe entre la formación que les damos y lo que impera en la práctica escolar, que discurre muchas veces por caminos bien distintos.

Es curioso, sin embargo, que pese al anacronismo que el modelo tradicional plantea con los tiempos que corren, donde los jóvenes están en permanente contacto y dependencia con las nue- vas tecnologías, lo que se observa en la realidad concuerda en ocasiones con la forma de concebir la enseñanza que tienen los futuros maestros, tal como revelan muchas veces con sus opiniones o con sus acciones. Quizás por la influencia que sobre ellos ejerce el modelo de enseñanza tradicional que la mayoría han vivido en sus años de escolarización, su idea de cómo debe ser el proceso de enseñanza se centra más en los conocimientos que deben adquirir los alumnos que en su proceso de aprendizaje. Al menos eso es lo que dice nuestra experiencia: cuando en segundo curso del Grado les pedimos que diseñen una propuesta de intervención en el aula utilizando metodologías activas y planteando actividades de desarrollo no llegan al $10 \%$ los que en un primer boceto del proyecto consiguen salirse del modelo expositivo donde el tipo de actividades planteadas son solamente de evaluación. Y es que, a pesar de que entienden la teoría que fundamenta esta metodología y comparten, según nos confiesan, los principios en los que se basa, llegar a diseñar estrategias para ponerla en práctica entraña para ellos una verdadera dificultad porque supone romper con sus propios preconceptos sobre lo que es la enseñanza.

Esto concuerda con los resultados de un estudio sobre los manuales escolares de Ciencias Sociales y en el que se pedía a los futuros maestros elegir el modelo de manual escolar preferente entre el de los años sesenta, el de los ochenta o el actual (Parra, 2010): si bien la práctica totalidad de los alumnos rechazaba el 
modelo descriptivo y basado en aprendizajes memorísticos que representaban los de los años 60, con respecto a los otros dos, se comprobó que eran más los estudiantes partidarios del manual de los años ochenta (con un sesgo materialista histórico claro) que del actual (mucho más influenciado por la historia de las mentalidades o la nueva historia sociocultural).

$\mathrm{Su}$ posicionamiento varía, sin embargo, cuando se les pide opinión, no ya sobre los contenidos disciplinares que se enseñan sino, sobre los modelos de enseñanza en las aulas escolares. Ante dos supuestos modelos de profesor de Ciencias Sociales descritos en la obra de Santisteban y Pagès (2011, pp. 23-24), uno de ellos más tradicional, centrado en conseguir que sus alumnos memoricen datos e información relevante sobre nuestra Historia, y el otro más innovador, dirigido a la reflexión en torno a los elementos que definen nuestro actual sistema social y democrático y a comprometer al alumnado en la mejora social, nuestros alumnos del Grado de Primaria valoran muy positivamente, y de forma unánime, al segundo, que toma decisiones democráticamente en el aula, hace salidas con sus escolares, si bien, en coherencia con lo que señalábamos más arriba, consideran de gran importancia los objetivos que persigue el primero ("desarrollar un amor por la historia y la geografía") y valoran los recursos (mapas de todo tipo, documentos históricos y geográficos...) y las actividades que plantea para ello (murales, redacciones, reali- zación de dibujos y maquetas...). Curiosamente, sin embargo, cuando son ellos mismos los que se ven implicados como estudiantes en un proceso innovador de enseñanza que rompe la estructura tradicional, confiesan sentirse perdidos, atastacados... y de algún modo, "al borde del caos", tal como ha demostrado una invetigación reciente sobre las opiniones manifestadas por estudiantes universitarios de PBL en las reuniones tutoriales (Barret, 2010).

Contexto de renovación metodológica en el que nace nuestra propuesta

Algunos años antes de la implantación del Plan Bolonia ya se venían desarrollando en la Universidad de Valladolid (UVA) proyectos de innovación encaminados a la Renovación de las Metodologías docentes en el marco de la Convergencia Europea hacia el EEES. Precisamente al amparo del Proyecto que, con este mismo título, fue concedido a esta Universidad por la Agencia para la Calidad del Sistema Universitario de Castilla y León (curso 20072008) se publicaron las diferentes experiencias que en este sentido estaban desarrollando profesores de la UVA de distintas titulaciones (Guilarte, 2008). En la Escuela de Educación de Palencia (Facultad desde el 5 de diciembre de 2013), este espíritu renovador se hizo patente ya en el curso 2005-2006 en que asumió el reto de comenzar a desarrollar un Proyecto Piloto para la implantación de Experiencias de Innovación docente para el EEES en el que dos cur- 
sos más tarde (2007-2008) participaba ya todo el profesorado del Centro.

La reformulación de los contenidos a enseñar y de la metodología docente que exigía el trabajo por competencias se había iniciado, pues, tres cursos antes de que arrancara en Palencia, y en el conjunto de las Universidades españolas, la primera promoción de estudiantes de los Grados de Educación Infantil y Educación Primaria sujeta al Plan Bolonia (2009-2013). En esta reformulación había participado activamente el área de Didáctica de las Ciencias Sociales, cuyas profesoras formaban parte, además, de un Grupo de Trabajo Interuniversitario que, constituido en 2005-2006 y bajo la dirección de una de ellas, la profesora Sánchez Agustí, elaboró diferentes propuestas para la formación del Maestro de Primaria (2006) y de Infantil (2007) unificando los esfuerzos de profesores de la Universidad de Burgos, Oviedo, País Vasco y Valladolid (Blanco et al., 2007).

Pero el compromiso que entonces se asumía desde nuestra área se correspondía, además, con los esfuerzos renovadores que se venían haciendo a nivel nacional desde el campo específico de la Didáctica de las Ciencias Sociales donde, aún hoy, sigue vigente la demanda que en 2001 realizara el profesor Prats sobre la necesidad de realizar investigaciones que profundicen en el "diseño, experimentación y evaluación de estrategias didácticas y la creación de materiales y elementos que sirvan para intervenir de manera controlada en el proceso de enseñanza y aprendizaje, (...) en profunda relación e interacción con la praxis" (Prats y Valls, 2011, p. 26). Y precisamente en esta línea se sigue enmarcando nuestra participación en diversos proyectos de innovación docente dentro de nuestra Universidad como son, en la actualidad, el de "Experiencia transdisciplinar de aprendizaje vivencial en la formación de educadores y educadoras" y el de "El Practicum como eje de desarrollo formativo y profesional en los Grados de Educación".

En definitiva, es en este contexto de renovación, y constante revisión, de la metodología docente que viene haciendo el profesorado de Didáctica de las Ciencias Sociales, en la Facultad de Educación de Palencia, y también en otros centros universitarios, donde se explica nuestra propuesta interdisciplinar de Aprendizaje Basado en Proyectos para segundo curso del Grado de Primaria. Ésta nace, por tanto, con una voluntad de debate en torno a nuevas ideas que contribuyan al desarrollo de metodologías inductivas en la enseñanza universitaria de la Didáctiva de las Ciencias Sociales con las que promover la adquisición, por parte de los alumnos, de las competencias profesionales del Maestro.

\section{Objetivos de la propuesta}

La propuesta de innovacción metodológica que aquí presentamos, la cual se articula en torno al Proyecto Didáctico que, entre las tareas no presenciales, pedimos a nuestros alumnos en la asignatura de Didáctica de las Ciencias Sociales en el Grado de Primaria, 
pretende abordar la enseñanza de este campo de conocimiento específico desde los planteamientos metodológicos del Aprendizaje Basado en Proyectos. Aunque al hilo del discurso previo entorno al contexto en que surge esta propuesta metodológica ya hemos introducido algunas de nuestras intenciones, es preciso hacer explícitos los objetivos que con ella nos proponemos alcanzar:

- Colaborar, desde la formación de maestros, en la renovación metodológica de la escuela, en particular en la etapa de Educación Primaria, donde desde diferentes sectores demandamos una mayor implicación de los escolares en su propio proceso de aprendizaje.

- Contribuir al debate y la reflexión en torno a la renovación de la metodología de enseñanza que nos exige el EEES a través del planteamiento y desarrollo de un posible modelo de enseñanza de la Didáctica de las Ciencias Sociales en el Grado de Primaria que favorezca en el alumno aprendizajes más significativos y más útiles para su desarrollo profesional.

- Generar entre el alumnado aprendizajes significativos en torno a la Didáctica de las Ciencias Sociales a partir de su implicación directa en el diseño de un proyecto educativo que parta de la cotidianidad y que sea susceptible de ser desarrollado en las aulas de E. Primaria.

- Poner en valor el carácter relacional y polivalente que poseen los contenidos específicos de las Cien- cias Sociales para proponer modelos globalizadores de enseñanza en Primaria que permitan establecer relaciones entre una variedad de disciplinas (no solo las Sociales) con el fin de explicar la realidad atendiendo a su complejidad.

- Favorecer entre el alumnado el aprendizaje colaborativo, donde el intercambio de ideas y propuestas se revela una vía para el enriquecimiento personal y profesional; así como el desarrollo de un espíritu reflexivo y crítico, para valorar la riqueza y utilidad de los planteamientos y materiales didácticos propios y de otros; y del conjunto de las competencias básicas que las leyes de educación exigen a los ciudadanos.

\section{Propuesta de aprendizaje basado en proyectos en el Grado de Primaria}

Desde la asignatura de Didáctica de las Ciencias Sociales tratamos de preparar a nuestros estudiantes de $2^{\circ}$ curso del Grado de Primaria por un aldo, para que sepan analizar intervenciones que desarrollan actualmente en las aulas maestros de Primaria para enseñar contenidos sociales; y por otro, para que, con autonomía, rigor y creatividad, sepan compartir ideas y cooperar entre sí para planificar y desarrollar propuestas de intervención en el aula de Primaria que utilicen metodologías de enseñanza activa. 
Como entendemos que la escuela debe facilitar al alumno ese ir y venir entre la realidad, que es el contexto en que se desenvuelve, y cada una de las disciplinas que contribuyen a explicarlo, estableciendo esa dialéctica necesaria entre contextualización y descontextualización que desde hace ya veinte años se viene demandando (Rozada, 1994, p. 46), pedimos a nuestros futuros maestros que diseñen su propuesta de intervención desde un enfoque globalizador, y en torno a un problema socialmente relevante (Benejam, 1999) donde el único documento-guía sea el texto legal que define el currículo de la etapa. Como hasta el momento, en que no se han aplicado en las aulas los Decretos de aplicación de la LOMCE, el área de Conocimiento del Medio, en el que se han venido desarrollando los contenidos sociales, integraba también contenidos propios de las Ciencias Experimentales, y puesto que apostamos, como decimos, por propuestas globalizadoras que permitan a los escolares entender la realidad tal como es, planteamos el proyecto de forma coordinada con la asignatura de Desarrollo Curricular de las Ciencias Experimentales, que se imparte también en $2^{\circ}$ curso de Primaria y en la que, por tanto, están matriculados prácticamente los mismos alumnos.

\section{Planteamiento del proyecto}

El planteamiento del proyecto se hace a partir de la semana 5 del curso y surge a partir de una pregunta motriz con la que pretendemos hacer al proyecto desafiante y conectarlo al tiempo con la realidad. Un ejemplo sería: ¿puede un niño de segundo ciclo de primaria aprender la geografía física de España si no le explico previamente la hidrografía y el relieve?. A partir de la pregunta, que tiene diferentes posibles respuestas surge un debate en el aula que nos lleva a hacer el enunciado del proyecto, en que planteamos a los estudiantes un problema creíble que puede darse en su futuro ámbito profesional. Algunos ejemplos podrían ser:

- Una nueva editorial de textos y materiales educativos que pretende desarrollar metodologías activas en el aula de Primaria está buscando especialistas en el área de Conocimiento del Medio. El objetivo es elaborar una propuesta didáctica innovadora para que los alumnos de segundo ciclo aprendan los principales formas de relieve y accidentes geográficos de la Península Ibérica de forma inductiva y por tanto, sin facilitarles previamente la información, sino trabajando estos contenidos dentro de otros ejes temáticos más amplios que se abordan también en ese ciclo. ¿Qué eje temático utilizarías tú? ¿en qué consistiría tu propuesta?

- Trabajas en un centro de Primaria que se ha abierto hace dos años y cuya seña de identidad es el aprendizaje cooperativo. El próximo año empieza tercer curso la primera promoción, y te encargan que elabores una propuesta de la Progra- 
mación General Anual del área de Conocimiento del Medio y que coordines las actuaciones que pueden hacerse de forma interdisciplinar para favorecer el conocimiento del alumno de la geografía física de España. ¿Cuál sería tu propuesta?

El proyecto se desarrollará en grupos de 3-4 estudiantes que se unen de forma libre y especifican en ese momento, en una carta de constitución del grupo que entregan al profesor, los momentos concretos de la semana que dedicarán a reunirse fuera del horario lectivo. Este documento es especialmente importante para nuestros alumnos, que adquieren un compromiso entre ellos mismos para sacar adelante el proyecto, y para nosotros, que nos aseguramos de que las horas no presenciales que contemplan los ECTS se cumplen. Y es que éste es un proyecto extenso que exige una importante dedicación: 168 horas (7 horas por alumno a la semana durante las siete que dura el proyecto) que ellos mismos han de saber organizar, computar y registrar en un documento personal y en las actas de las reuniones que realicen semanalmente, según lo que han establecido en esa carta de constitución.

Pero no es solo el tiempo que dedicamos al proyecto (semanas 6-13 del curso) lo que define su carácter extenso, y no reducido, sino todo el planteamiento del mismo: se articula en torno al eje temático que decide el propio grupo -de común acuerdo con el profesor y no por imposición nuestra-, las fases para su desarrollo son definidas mayoritariamente por los propios miembros del grupo, y además dentro de éste es importante el debate para la toma de decisiones, dada la multitud de variables que pueden tomar en cuenta para elaborar su propuesta.

Criterios de calidad: cumplimiento de compromisos y de objetivos

Los criterios de calidad del proyecto se concretan a partir de dos elementos: 1) el cumplimiento de los compromisos asumidos por cada grupo en cuanto a las fases del desarrollo del proyecto; 2) el grado de consecución de los objetivos didácticos que planificamos para el mismo y, los cuales, con independencia del enunciado concreto que tenga el proyecto, vienen a ser siempre más o menos los mismos:

- Justificar adecuadamente una propuesta de intervención en base al Real Decreto que regula las enseñanzas mínimas de la etapa educativa para la que se preparan como maestros.

- Diseñar propuestas de intervención educativa en el aula de primaria para abordar contenidos-problemas sociales relevantes en Primaria donde apliquen los principios psicopedagógicos y didácticos sobre la enseñanza y el aprendizaje del espacio geográfico y del tiempo histórico pero donde tengan cabida también, por su enfoque globalizador, otras áreas de conocimiento de la etapa (Prats, 2000).

- Definir en su propuesta didáctica los diferentes elementos que inter- 
vienen en una programación: competencias, objetivos y contenidos, metodología, temporalización, actividades de desarrollo, recursos didácticos, criterios (y estándares de aprendizaje) y sistemas de evaluación.

- Diseñar y elaborar los materiales didácticos necesarios para desarrollar los contenidos programados en el proyecto, tanto si son planificados para que los realice el maestro como si se integran dentro de actividades que han de hacer los escolares.

Seguimiento del Proyecto: hitos y dificultades

Una semana después de la constitución de los grupos (semana 7) todos ellos han de entregar un primer boceto de su propuesta de intervención, donde quede definido el eje temático sobre el que articularán su propuesta, los objetivos y contenidos del currículo oficial para el área de Conocimiento del Medio que van a abordar, y el primer esbozo de al menos tres de las cinco actividades de desarrollo que plantean en su propuesta. El objetivo es conocer las propuestas iniciales de nuestros alumnos para poder reorientarles si es necesario. Las siguientes fases en el desarrollo del proyecto las definen ellos mismos estableciendo un cronograma que tendrán que entregarnos en la semana 8.

Esta segunda entrega es un documento muy importante para los alumnos porque les obliga a organizarse no dejando todo para el último momento $\mathrm{y}$ a cumplir los plazos que ellos mismos establecen para cada hito. Pero también lo es para el profesor porque nos revela la capacidad del grupo para establecer acuerdos y organizarse, la pertinencia de los hitos establecidos por el grupo y los momentos que podemos requerirles en tutoría no solo para comprobar el cumplimiento de los mismos sino, sobre todo, para orientarlos y acompañarlos, si se estima necesario, en la toma de decisiones.

$\mathrm{Y}$ es que, con frecuencia, es preciso intervenir para que la propuesta sea globalizadora y plantee el aprendizaje como una elaboración activa por parte de los escolares de primaria. Las actividades que proyecten han de ser, como decíamos antes, de desarrollo y deben perseguir, igual que ocurre en los proyectos de trabajo, que el alumno construya su aprendizaje según su propia experiencia, y que el maestro, en vez de ser el transmisor de los conocimientos, se convierta en la persona que guía y orienta, provoca conflictos y ayuda a la búsqueda de soluciones durante todo el proceso (Muñoz y Díaz, 2009; Pozuelos, 2007). Y he aquí uno de los principales escollos que para muchos de nuestros alumnos tiene este trabajo: la distinción entre actividades de desarrollo y actividades de evaluación.

Normalmente, y quizás, como decíamos más arriba, por los modelos de enseñanza que ellos mismos han conocido en su escolaridad, las actividades que plantean son lúdicas, sí, pero del segundo tipo, encaminadas a valorar aprendizajes previos o adquiridos tras una 
intervención (adaptaciones del juego de la oca, trivial...). Que entiendan la diferencia y sepan diseñar ambos tipos de actividades es, sin embargo, fundamental -y por eso esta parte del trabajo tiene especial relevancia-, porque pone al descubierto su comprensión de lo que es una metodología activa y participativa así como el grado de coherencia entre la planificación y la ejecución del proyecto.

Exposición pública de los resultados del proyecto

Los resultados del proyecto se exponen ante toda la clase en torno a la tercera y segunda semana antes de finalizar el período lectivo, facilitándonos así un nuevo instrumento para la valoración del trabajo que desarrolla cada grupo.

Aunque su propuesta de intervención no se llega a desarrollar en un aula real de Primaria los estudiantes sí que ponen en práctica al menos tres de las actividades que proponen en su trabajo ante sus compañeros de aula en la Universidad, y contando además con ellos, que por un momento y de forma algo forzada "se transforman" en alumnos de Primaria. Todos los estudiantes escuchan y participan de las propuestas de todos, y a partir de unas fichas de registro y valoración, no solo hacen una evaluación del trabajo propio (ficha de registro-reflexión-autoevaluación del propio trabajo) y de los del resto (ficha de registro-valoración de trabajos de los compañeros), sino que obtienen un pequeño "catálogo" de propuestas de intervención en el aula de Primaria.

\section{Resultados y valoración de la propuesta}

Para la valoración de esta propuesta de ABP utilizamos diferentes informaciones que obtenemos durante el proceso de elaboración de los proyectos didácticos, con los bocetos que nos entregan los alumnos y la discusión en torno a los mismos en tutorías y seminarios, y una vez que éstos ya son entregados, donde al tiempo que terminamos de evaluar el cumplimiento de los objetivos, su factura técnica y su exposición a los compañeros, conocemos las valoraciones y opiniones que en torno al mismo vierten sus autores. Y es que, en la estructura del trabajo, los alumnos han de incluir un apartado de valoración del proceso seguido para su desarrollo en lo referente, por un lado, a su propia propuesta y las dificultades encontradas para definirla y, por otro lado, al grupo de trabajo, a su funcionamiento y al propio proceso de aprendizaje que han vivido como tal, desde sus ideas iniciales hasta el diseño y desarrollo final por escrito de su Proyecto Didáctico. En esa valoración, que es libre en cuanto a que no ha de sujetarse a ningún item concreto, salen a flote reflexiones de gran interés para nosotros, pues permiten confirmar nuestras propias impresiones como profesora y, sobre todo, las potencialidades que para el alumno ofrecen este tipo de planteamientos en el aula.

Un dato esperanzador es que, desde que en el curso 2011-2012 pusimos en marcha esta propuesta de trabajo, ningún grupo ha valorado negativamente 
esta experiencia. A pesar de las dificultades que, confirmando lo señalado por Egido y colaboradores (2007) en cuanto a las limitaciones del ABP, algunos estudiantes confiesan, de forma explícita, haber tenido para "arrancar", "organizarse" o "encontrar el equilibrio en lo que creo que los niños son capaces de comprender y lo que no", o de la "gran cantidad de trabajo que supone", todos los grupos señalan algo positivo que les ha aportado el trabajo. Bien porque les permite aprender más y mejor los contenidos de nuestra asignatura e incluso de otras que tienen a lo largo de la Titulación, bien porque les ayuda a organizar ideas para llevarlas a la práctica en el aula, pensar la enseñanza de otro modo... su balance final es positivo y encontramos con frecuencia frases del tipo "en líneas generales estamos bastante satisfechos con el trabajo" (G-G-C, 2012-2013). Este mismo grupo continúa además diciendo "el trabajo realizado nos ha permitido ponernos en mayor contacto con la asignatura y tener así una mayor comprensión de sus contenidos. También nos ha puesto en contacto con la preparación de un programa por proyectos, algo que, hasta ahora, apenas habíamos tocado. El resultado de este contacto es sin duda interesante pues nos permite ver desde una visión más amplia las diferentes metodologías de enseñanza".

Algunos grupos valoran especialmente el hecho de que el trabajo les ha ayudado reforzar los hábitos de trabajo en grupo, que no es una suma de partes sino como una vía de intercambio y colaboración: "Este proyecto... ha consolidado nuestro modo de trabajar en grupo, algo a lo que no estábamos muy acostumbradas antes de empezar con este Grado. (...) La mayor riqueza de este trabajo, no sólo son las risas que hemos compartido, sino que hemos sabido sustraer lo mejor de cada una de nosotras, es decir, que cada una hemos aportado nuestras capacidades por el bien del grupo, ya que eso es lo más jugoso e interesante del trabajo en equipo, aprender de y con los otros ("aprendizaje social", Vygotsky). Reflexionando sobre nuestros puntos fuertes y débiles nos hemos percatado de que nunca habíamos pensado en ese tipo de cosas, ya que nunca nos habíamos autoevaluado como grupo (...)" (AC-IC, 2011-12).

Otras opiniones se centran en valorar positivamente las posibilidades que ofrece en el aula el enfoque globalizador de la enseñanza: "El mayor aprendizaje que he adquirido es el de la cantidad de posibilidades que se pueden abordar desde un tema en esta área de Conocimiento del Medio. Mismamente, mediante los conflictos se pueden trabajar muchos contenidos actitudinales y conceptuales referidos a la resolución de conflictos y a grandes conflictos de la Historia; pero también pueden trabajarse aspectos del espacio mediante planos, del tiempo con cronogramas, de expresión oral en debates, de expresión escrita en manifiestos o descripciones de los hechos, aspectos creativos, de autonomía, de formación del sentido crítico, matemáticos (existen multitud de cifras en cualquier guerra), y un largo etcétera" (AV, 2012-13). En este senti- 
do les resulta positiva la exigencia de tomar en cuenta la realidad cotidiana de los escolares y sus propios intereses para diseñar su proyecto como garantía de un aprendizaje: "El principal punto fuerte del proyecto creo que reside en la originalidad y en la posibilidad de tratar un tema tan complejo para niños de Educación Primaria desde situaciones que les rodean casi a diario. (¿Quién no ha tenido disputas por el campo de fútbol en el recreo?)" (AV, 2012-2013); "Este proyecto nos ha permitido saber cómo podemos elaborar toda una propuesta de intervención partiendo de los intereses del alumnado. Creemos que partiendo de un enfoque globalizador el alumno puede comprender la realidad como un todo, y no como contenidos compartimentados que posiblemente no lleguen a hilar nunca. Por medio del método de proyectos, el alumno tiene posibilidad de desarrollar un aprendizaje significativo, construyendo sus conocimientos poco a poco y sobre todo tomando como punto de partida sus propios intereses sobre la realidad. En este sentido, el alumno adquiere unos conocimientos con una fuerte carga emocional, por lo que existe una mayor probabilidad de que estos permanezcan durante más tiempo en su estructura cognitiva (...)." (I-M-N, 2012-13).

En definitiva, los resultados de la plicación de esta metodología de enseñanza, tanto por la calidad de los trabajos presentados, bien justificados, documentados, y de gran riqueza y originalidad no solo en su diseño y los materiales aportados sino también en su expo- sición (implicando a los compañeros en una representación teatral, una jimkana, un baile regional, un comercio solidario...), como por las propias opiniones manifestadas por los estudiantes, permiten confirmar las ventajas que, como decíamos en nuestra introducción, se han atribuido a este modelo: los estudiantes obtienen un conocimiento más profundo de la materia y de los problemas profesionales que plantea su puesta en práctica en el aula, y mientras desarrollan estrategias para obtener y analizar información que les ayude a resolverlos, dan sentido y utilidad a sus propios aprendizajes, lo cual a su vez despierta en ellos un mayor interés por aprender, no solo individualmente sino sobre todo de forma cooperativa, permitiendo que el proceso de aprendizaje se retroalimente continuamente.

\section{Conclusiones}

A través de nuestra propuesta para aplicar el ABP en las aulas universitarias del Grado de Primaria creemos estar más cerca de conseguir un doble objetivo: por un lado, que nuestros futuros maestros comprendan de manera vivencial (a través de su propia experiencia de $\mathrm{ABP}$ ) y, en consecuencia valoren positivamente, lo que aportan al discente las metodologías de enseñanza activa, y por otro lado, que sean capaces de utilizarlas para diseñar propuestas de intervención globalizadoras susceptibles de ser aplicadas en las aulas de Primaria.

En los trabajos por proyectos que se desarrollan en las escuelas los niños y 
las niñas ya sean de la etapa Infantil o de Primaria, realizan en torno a un centro de interés explícito o implícito diferentes propuestas para investigar y experimentar, organizan entre todos un plan de actuación y deciden, junto con el maestro, los pasos a dar para finalmente llegar a unas conclusiones. Algo parecido es lo que hacemos nosotros con nuestros estudiantes universitarios, pero nuestro centro de interés es la propia práctica docente, sobre la que reflexionan e investigan como futuros profesionales de la enseñanza. Respecto a ésta, no les podemos pedir que diseñen un trabajo por proyectos tal como se desarrolla en un aula de Primaria, pues nos faltan los escolares, que son la pieza clave de los proyectos, pero sí que partan en su propuesta de intervención de la misma concepción constructivista. Y eso sí que lo logramos en la práctica totalidad de los casos (apenas contamos con suspensos en estos trabajos, quizás por el seguimiento que hacemos de los mismos), y en muchos de ellos con un alto grado de satisfacción. Pese al esfuerzo que este tipo de planteamientos exige a los alumnos (tiempo de dedicación, coordinación entre varios miembros, búsqueda de información, rigor para la justificación de su proyecto, coherencia y creatividad para su diseño, habilidad para la elaboración de materiales...) el balance que ellos mismos realizan resulta muy positivo: son conscientes de las fases que han atravesado en el proceso, de su propia implicación y la del resto de sus compañeros de grupo, aprenden a autocorregirse individual y grupalmente, reforzando y ampliando, además, sus propios aprendizajes en torno a la Didáctica de las Ciencias Sociales.

Pero, junto a los resultados del proyecto en sí, igualmente interesante para nosotros es que después de esta experiencia nuestros alumnos encuentran diferentes modelos de referencia para aplicar en su futuro profesional este tipo de metodologías: el proyecto que cada uno desarrolló con su grupo, los de los otros grupos de su clase y la propia experiencia que como alumnos tuvieron en nuestras aulas. Muchos de nuestros estudiantes deciden, de hecho, en el curso siguiente, durante el Practicum I, poner en práctica el proyecto que su grupo u otros compañeros diseñaron en nuestra asignatura, o hacer alguna de las actividades que nosotros mismos desarrollamos en el aula universitaria. Aunque esta decisión depende fundamentalmente de si su contenido encaja o no con los que se están trabajando en el colegio donde desarrollan sus prácticas, el mero hecho de poder plantearse su aplicación nos hace pensar que estamos cerca de lograr esa dialéctica necesaria entre el aula y la realidad.

Sin duda, es preciso seguir revisando nuestra práctica docente universitaria en las Facultades de Educación y apostar de forma más decida, uniendo esfuerzos entre el profesorado, porque los futuros maestros otorguen en su desarrollo profesional el protagonismo al alumnado poniendo en valor el proceso de enseñanza y no solo el resultado. En definitiva, creemos que desde las aulas 
universitarias donde se forman nuestros maestros debemos seguir desarrollando este tipo de metodologías de enseñanza para poder contribuir a la formación de maestros reflexivos en torno a la práctica docente, críticos ante la inercia en el modo de enseñar, comprometidos con las necesidades educativas que para los alumnos plantea el contexto social en que se integran, y creativos para innovar en su entorno profesional y mejorar nuestro sistema educativo.

\section{Referencias bibliográfícas}

BARRETT, T. (2010). The problembased learning process as finding and being in flow. Innovations in Education and Teaching International, vol. 47, $\mathrm{n}^{\circ} 2,165-174$. DOI: $10.1080 / 14703291003718901$.

BARRETT, T. (2005). Understanding problem-based learning. En T. BARRETT, I. MACLABHRAINM, H. FALLON (eds.) Handbook of enquiry \& problem based learning (13-25). Galway: CELT. Recuperado de <http:// www.nuigalway.ie/celt/pblbook/chapter2. pdf $>$ [5 de mayo de 2014].

BENEJAM, P. (1999). La oportunidad de identificar conceptos clave que guíen la práctica curricular de las ciencias sociales. Iber. Didáctica de las Ciencias Sociales, $\mathrm{n}^{\circ}$ 21, 8-10.

BLANCO, P.; DE LA CALLE, M. et al. (2007). La implantación del European Credit Transfer System en los contenidos de Didáctica de las Ciencias Sociales del Título de Maestro en Educación Infantil. En C. GUILARTE (Coord.) Experiencias de Innovación Docente de la Universidad de Valladolid (189-200). Valladolid: Universidad de Valladolid.

CUBERO, R. (2005). Elementos básicos para un constructivismo social. Avances en psicología latinoamerica$n a$, vol. 23, $\mathrm{n}^{\circ} .1,43-61$. Recuperado de $<$ http://revistas.urosario.edu.co/index. php/apl/article/view/1240> [14 de mayo de 2014].

EGIDO,I.,ARANDA, R., CERRILLO,R., DE LA HERRÁN, A., DE MIGUEL, S., GÓMEZ, M., HERNÁNDEZ, R., IZUZQUIZA, D., MURILLO, F.J., PÉREZ, M. \& RODRÍGUEZ, R.M. (2007). El aprendizaje basado en problemas como innovación docente en la universidad: Posibilidades y limitaciones. Educación y Futuro, $\mathrm{n}^{\mathrm{0}}$ 16, 85-100.

FALAIZE, B. (2010). El método para el análisis de los temas sensibles de la historia. En R. ÁVILA, M.P. RIVERO Y P.L. DOMÍNGUEZ (eds.) Metodología de investigación en didáctica de las Ciencias Sociales (187-205). Zaragoza: IFC-AUPDCS.

GARRIGÓS SABATÉ, J.; VALEROGARCÍA, M. (2012). Hablando sobre Aprendizaje Basado en Proyectos con Julia. Revista de Docencia Universitaria. REDU, vol.10 (3), 125151. Recuperado de <http://www. red-u.net/> [25 de mayo de 2013].

GAVALDÀ, A., PONS I ALTÉS, J.M. (2010). La práctica competencial a partir de las Ciencias Sociales. 
En R. ÁVILA, M.P. RIVERO Y P.L. DOMÍNGUEZ (eds.) Metodología de investigación en didáctica de las Ciencias Sociales (607-615). Zaragoza: IFC-AUPDCS

GUILARTE, C. (Coord.) (2008). Innovación docente: Docencia y TICs. Valladolid: Universidad de Valladolid.

MABROUK, P. A. (ed.) (2007). Active learning: models from the analytical sciences. Washington: American Chemical Society.

MUÑOZ, A; DÍAZ, M.R (2009). Metodología por proyectos en el área de conocimiento del medio. Revista Docencia e Investigación, $\mathrm{n}^{\circ}$ 19, 101-126.

PARCERISA ARAN, A. (2006). Plan docente: planificar las asignaturas en el marco europeo de Educación Superior. En VV.AA. Propuestas para el cambio docente en la Universidad (49-74). Barcelona: OctaedroICE-UAB.

PARRA, D. (2010). La importancia de las tendencias historiográficas en la concepción de la historia y su didáctica para futuros maestros. En R. ÁVILA, M.P. RIVERO Y P.L. DOMÍNGUEZ (eds.) Metodología de investigación en didáctica de las Ciencias Sociales (257-262). Zaragoza: IFC-AUPDCS.

POZUELOS, F.J. (2007). Trabajo por proyectos en el aula: descripción, investigación y experiencias. Sevilla: Cooperación Educativa.

PRATS, J. (2000). Disciplinas e interdisciplinariedad: el espacio relacional y polivalente de los contenidos de la Didáctica de las Ciencias Sociales. Iber. Didáctica de las Ciencias Sociales, Geografía e Historia, n ${ }^{\circ}$ 24, 7-17.

PRATS, J.; VALLS, R. (2011). La Didáctica de la Historia en España: estado reciente de la cuestión. Didáctica de las Ciencias Experimentales y Sociales, n. ${ }^{\circ}$ 25, 17-35. Recuperado de < http://attic.uv.es/index.php/dces/article/ view/2360/1916> [20 de abril de 2014].

PRIETO,A., BARBARROJA, J., REYES, E.L., MONTSERRAT, J., DÍAZ, D., VILLAROEL, M. \& ÁLVAREZ-MON, M. (2006). Un nuevo modelo de aprendizaje basado en problemas, el $\mathrm{ABP} 4 \mathrm{x} 4$, es eficaz para desarrollar competencias profesionales valiosas en asignaturas con más de 100 alumnos. Aula Abierta, n ${ }^{\circ} 87,171-194$.

PRINCE, M.J.; FELDER, R.M. (2006) Inductive Teaching and Learning Methods: Definitions, Comparisons, and Research Bases. En J. Engr. Education, 95(2), 123138. Recuperado <http://www4.ncsu. edu/unity/lockers/users/f/felder/public/ Papers/InductiveTeaching.pdf $>$ [28 de mayo de 2013].

RAMSDEM, P. (2003). Learning to teach in higher education. London; New York: RoutledgeFalmer.

ROZADA MARTÍNEZ, J.M. (1994). Los contenidos en la enseñanza de las Ciencias Sociales: la opción disciplinas-problemas relevantes. En Grupo Ínsula Barataria (coord.) Enseñar y aprender Ciencias Sociales: algunas propuestas de modelos didácticos 
(31-54). Madrid: Mare Nostrum Ediciones Didácticas.

SANTISTEBAN, A., PAGÈS, J. (coords.) (2011). Didáctica del Conocimiento del Medio Social y Cultural en Educación Primaria. Ciencias Sociales para comprender, pensar y actuar. Madrid: Síntesis.

SEVA, F; SORIANO, M.C y VERA, M.I. (2010). La práctica docente en las Ciencias Sociales: Un análisis cualitativo. En R. ÁVILA, M.P. RIVERO Y P.L. DOMÍNGUEZ (eds.) Metodo- logía de investigación en didáctica de las Ciencias Sociales (175-183). Zaragoza: IFC-AUPDCS.

SOLAZ-PORTOLÉS. J.J.; SANJOSÉ, V. y GÓMEZ, A. (2011). Aprendizaje basado en problemas en la Educación Superior: una metodología necesaria en la formación del profesorado. Didáctica de las Ciencias Experimentales y Sociales, $\mathrm{n}^{\circ} 25,177-186$. Recuperado de <https://ojs.uv.es/index. php/dces/article/view/2369/1925> [20 de abril de 2014]. 
